\title{
Low Serum Free Triiodothyronine Is Associated with Increased Risk of Decompensation and Hepatocellular Carcinoma Development in Patients with Liver Cirrhosis
}

\author{
Ula M. Al-Jarhi1 ${ }^{*}$, Abeer Awad1, Mona Mohsen² \\ ${ }^{1}$ Department of Medicine, Cairo University, Cairo, Egypt \\ ${ }^{2}$ Department of Chemical Pathology, Cairo University, Cairo, Egypt \\ Email: ulamabid.aljarhi@gmail.com
}

Received 23 April 2016; accepted 6 June 2016; published 9 June 2016

Copyright (C) 2016 by authors and Scientific Research Publishing Inc. This work is licensed under the Creative Commons Attribution International License (CC BY). http://creativecommons.org/licenses/by/4.0/

(c) (i) Open Access

\section{Abstract}

Background: FT3 levels in plasma may provide a marker for liver status in cirrhosis. Aim: The aim is to correlate thyroid functions with hepatic status in compensated and decompensated cirrhosis, and to study their effect on development of HCC. Settings and Design: Prospective controlled cohort study. A total of 58 patients with liver cirrhosis were recruited from Kasr AlAiny ER and outpatient clinics. Patients were categorised into compensated (11), decompensated (39) and patients with hepatocellular carcinoma (8). The study also included 12 healthy controls. Methods and Material: Liver function tests, TSH, FT4 and FT3 and abdominal ultrasound and triphasic computed tomography abdominal scans were done. Statistical Analysis Used: Chi-square and unpaired t-tests were used for comparison. One way ANOVA and Kruskal Wallis tests were used to compare more than two groups. Spearman Correlation followed by logistic regression analysis of significant variables was used to find predictors of dependent variables. Results: The frequency of patients with low FT3 was significantly higher in patients with liver cirrhosis $(48 \%)$, and HCC $(50 \%)$ than control subjects $(12 \%)$ (p-value $<0.001)$. Mean serum FT3 was lowest among decompensated patients $(2 \mathrm{pg} / \mathrm{ml} \pm 0.7)$, followed by patients with $\mathrm{HCC}(2.5 \mathrm{pg} / \mathrm{ml} \pm 0.7)$ and highest among compensated patients $(3.7 \mathrm{pg} / \mathrm{ml} \pm 0.4)$, p-value $<0.001$. Logistic regression analysis showed that low FT3, male gender, ulcer bleeding and encephalopathy were independently associated with the development of HCC (OR, 95\% CI: 1.1, 0.3 - 8). Conclusions: Low FT3 is common among patients with decompensated liver cirrhosis and HCC. FT3 shows a significant negative

\footnotetext{
${ }^{*}$ Corresponding author.
}

How to cite this paper: Al-Jarhi, U.M., Awad, A. and Mohsen, M. (2016) Low Serum Free Triiodothyronine Is Associated with Increased Risk of Decompensation and Hepatocellular Carcinoma Development in Patients with Liver Cirrhosis. Open Journal of Gastroenterology, 6, 166-174. http://dx.doi.org/10.4236/ojgas.2016.66022 
correlation with severity of liver disease and deterioration of liver function. Low FT3 shows a significant independent association with HCC.

\author{
Keywords
}

Liver Cirrhosis, Thyroid Functions, HCC

\title{
1. Introduction
}

Thyroid hormones are general regulators of tissue metabolism; however, a unique relation with hepatocytes exists. The liver in turn metabolises thyroid hormones and regulates their systemic functions [1].

Low FT3 (free triiodothyronine) is the most frequent disruption encountered in routine screening of thyroid functions in patients with liver cirrhosis. This reflects a reduction in type 1 deiodinase activity, reduced T4 (thyroxine) conversion to T3 and compensatory shift to T4 conversion to rT3 (reversed T3) by type 3 deiodinase [2].

This reduction in FT3 levels may reflect a compensatory hypothyroid state in patients with liver cirrhosis that tunes down hepatocytes' metabolism which may help to preserve liver functions and conserve body protein stores [1]. The prognostic value of low FT3 in liver cirrhosis and its link to encephalopathy have been recognized for a long time [3]. Low serum T3 level is found to be an indicator of poor prognosis for hepatic encephalopathy [4]. However, it is seldom used in spite of its simplicity and low cost.

Overt hypothyroidism has been indirectly linked to HCC (hepatocellular carcinoma) on top of non-alcoholic steatohepatitis [5] [6]. In a previous study, a significantly elevated risk association is found between long term history of hypothyroidism and HCC in women [7]. However, a clear association of abovementioned thyroid derangement with HCC development has not been studied in patients with liver cirrhosis.

The aim of this work was to study to correlate thyroid functions with hepatic status in compensated and decopensated cirrhosis and to determine the effect of thyroid dysfunction in development of HCC.

\section{Methods}

\subsection{Subjects}

The study presents a prospective cohort. Patients with non-alcoholic liver cirrhosis, aged between 35 and 65 years were recruited from Kasr Alainy outpatient clinics over three months. Patients were consecutively recruited irrespective to the presenting symptom, hepatic status and presence of complications. Patients with history of head and neck irradiation, known (concurrent or past) extra-hepatic primary tumours or any type of primary liver cancer other than HCC, positive family history of cancer, or primary thyroid disorders were all excluded. A total of 58 patients with liver cirrhosis met the criteria and were included. Decompensated patients were admitted to wards of the internal medicine or to the intensive care according to admission protocols. Patients were followed up till discharge or mortality.

Liver function tests, TSH (thyroid stimulating hormone), FT4 and FT3 and abdominal ultrasound and triphasic computed tomography abdominal scans were done. Lipid profile was done to all patients. The patients were accordingly categorised into three groups: 39 patients with decompensated liver cirrhosis, 11 patients with compensated liver cirrhosis, 8 patients with HCC. The study included 12, age and gender matched healthy controls.

\subsection{Hormone Assays}

Hormone assays of TSH, FT3 and FT4 was carried out using electro-chemiluminescence immunoassay (ECLIA) on Cobas e411 (Roche Diagnostics International Ltd, Switzerland). Thyroid dysfunction was determined according to normal values by the manufacturer: TSH at $0.72-4.2 \mathrm{uiu} / \mathrm{ml}, \mathrm{FT} 4$ at $0.93-1.7 \mathrm{ng} / \mathrm{dL}$ and FT3 at 2.57 $-4.43 \mathrm{pg} / \mathrm{ml}$. classification of patients according to the thyroid profile was done following the guidelines of the National Academy of Clinical Biochemistry (NACB) for laboratory diagnosis and monitoring of thyroid diseases [8]. 


\subsection{Ethical Consideration}

A written informed consent was obtained from all participants or, if patients were unable to provide consent, from designated surrogates. Confidentiality of data, safe data storage and privacy rights are respected by all who handle patient information. Data was coded and patient names or identity was obscure in all data collection forms and during statistical analysis.

\subsection{Statistical Methodology}

Analysis of data was done by IBM computer using SPSS (statistical program for social science version 12) as follows: TSH, FT3 and FT4 were used both as continuous and categorical variables. Normal laboratory values were used to form categories for abnormal thyroid functions, e.g. "Low FT3", "High TSH". Description of quantitative variables as mean, SD and range. Description of qualitative variables was done as number and percentage. Chi-square test was used to compare qualitative variables between groups. Unpaired t-test was used to compare quantitative variables, in parametric data (SD $<50 \%$ mean). Mann Whitney Willcoxon U test was used in non parametric data instead of unpaired t-test. One way ANOVA (analysis of variance) was used to compare more than two groups as regard quantitative variable. Kruskal Wallis test was used instead of ANOVA test in non parametric data SD > 50\% mean. Spearman Correlation test was used to rank variables versus each other positively or inversely. Logistic regression analysis was used to find out the significant independent predictors of dependent variable by backward likelihood ratio technique. $\mathrm{p}$-value $>0.05$ was considered as insignificant, $\mathrm{p}$ $<0.05$ as significant and $\mathrm{p}<0.01$ as highly significant.

\section{Results}

As regards general data, all groups had similar age and gender distribution, which was also matching with the control group (Table 1).

The frequency of various presenting complications of liver cirrhosis was analysed in decompensated patients (Figure 1). SBP was the presenting complication in 5 patients, encephalopathy in 14 patients, GIT haemorrhage in 18 patients. Abdominal ultrasound revealed splenomegaly in all decompensated patients, and ascites in all but one.

Table 1. Comparison between the studied groups as regards socio-demographic characteristics.

\begin{tabular}{|c|c|c|c|c|c|c|}
\hline Variables & $\begin{array}{c}\text { Decompensated } \\
\mathbf{N}=39\end{array}$ & $\begin{array}{c}\text { Compensated } \\
\mathbf{N}=11\end{array}$ & $\begin{array}{l}\text { HCC } \\
\mathbf{N}=8\end{array}$ & $\begin{array}{c}\text { Controls } \\
\mathrm{N}=12\end{array}$ & $X^{2}$ a & p-value \\
\hline \multicolumn{7}{|l|}{ Gender } \\
\hline Male & 25 (66.7\%) & $6(54.5 \%)$ & $6(75 \%)$ & $8(66.7 \%)$ & \multirow{2}{*}{0.9} & \multirow{2}{*}{$\begin{array}{c}>0.05 \\
\text { NS }^{\mathbf{b}}\end{array}$} \\
\hline Female & 13 (33.3\%) & 5 (45.5\%) & 2 (25\%) & 4 (33.3\%) & & \\
\hline Age & $54.2 \pm 10$ & $50 \pm 11$ & $54 \pm 12.4$ & $49.5 \pm 11$ & 1.6 & $\begin{array}{c}>0.05 \\
\text { NS }\end{array}$ \\
\hline
\end{tabular}

${ }^{\mathrm{A}}$ chi-square test, ${ }^{\mathrm{B}}$ non-significant.

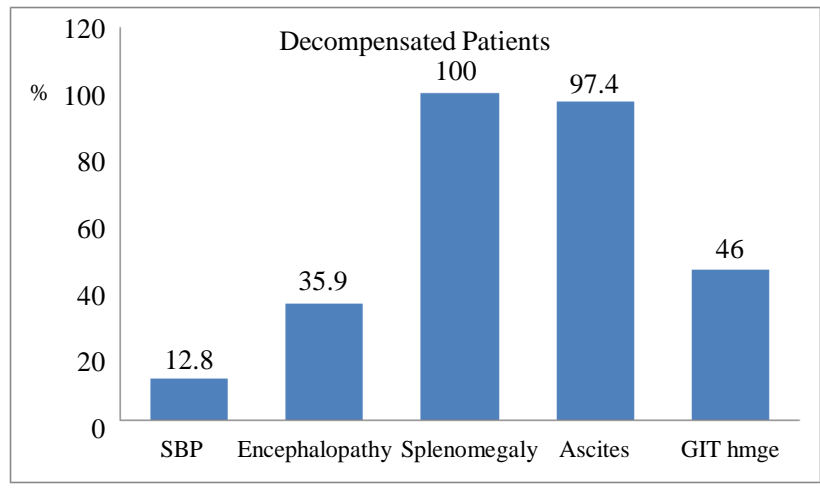

Figure 1. The frequency of various complications in decompensated patients. 
Table 2 shows that low FT3 was more frequent among patients with liver cirrhosis as compared to healthy controls with statistically significant difference by using chi-square test. Low FT3 was found in $48 \%$ of patients with liver cirrhosis and $50 \%$ of patients with HCC, as compared to only $12 \%$ in controls ( $p$-value $<0.001$ ).

Figure 2 shows that decompensated patients had the lowest level of FT3; while compensated patients had the highest level with statistically significant difference by using one way ANOVA test. Mean serum FT3 was lowest among decompensated patients $(2 \mathrm{pg} / \mathrm{ml} \pm 0.7)$, followed by patients with HCC $(2.5 \mathrm{pg} / \mathrm{ml} \pm 0.7)$ and highest among compensated patients $(3.7 \mathrm{pg} / \mathrm{ml} \pm 0.4)$. This difference was statistically significant, with $\mathrm{p}$-value $<$ 0.001 .

On comparing the relative frequencies of thyroid function categories among various groups, the prevalence of low FT3 was highest in decompensated patients (61.5\%), followed by HCC patients (50\%) versus none of the compensated patients (Figure 3). This difference was statistically significant with a p-value $<0.001$. As seen in Table 3, other thyroid function abnormalities were matching in frequencies with healthy controls.

On correlation of thyroid functions against other biochemical variables including liver functions and lipid profile, FT3 showed a significant direct correlation with albumin and prothrombin concentration (PC), while an inverse correlation with INR, Figure 4 and Figure 5 respectively.

In this study, FT4 level abnormalities were rare and statistically comparable to the control group. Only one patient with liver cirrhosis, hypoalbuminemia and ascites had low FT4. The patient also had low FT3. Elevated FT4 was present in one decompensated patient with low FT3, and one compensated patient with otherwise normal thyroid functions.

Backward likelihood technique of binary logistic regression analysis was done to test the effect of various factors on the incidence of each of the following complications of liver cirrhosis: ascites, SBP (spontaneous bacterial peritonitis), encephalopathy, GIT haemorrhage, HCC.

Table 2. Comparison between total group versus controls as regard thyroid profile.

\begin{tabular}{|c|c|c|c|c|c|}
\hline Variables & $\begin{array}{c}\text { Total Cases } \\
\mathrm{N}=50\end{array}$ & $\begin{array}{l}\mathrm{HCC} \\
\mathrm{N}=8\end{array}$ & $\begin{array}{c}\text { Controls } \\
\mathrm{N}=12\end{array}$ & $\mathrm{X}^{2}$ & $\mathrm{P}$ \\
\hline Low TSH & $5(10 \%)$ & $2(25 \%)$ & $1(8.3 \%)$ & 3.9 & $>0.05$ \\
\hline High TSH & $4(8 \%)$ & 0 & $1(8.3 \%)$ & 1.8 & $>0.05$ \\
\hline Low FT3 & $24(48 \%)$ & $4(50 \%)$ & 3 (12\%) & 25 & $<0.001$ \\
\hline Low FT4 & $1(2 \%)$ & 0 & 0 & 0.4 & $>0.05$ \\
\hline High FT4 & $2(4 \%)$ & 0 & $2(16.7 \%)$ & 3.2 & $>0.05$ \\
\hline
\end{tabular}

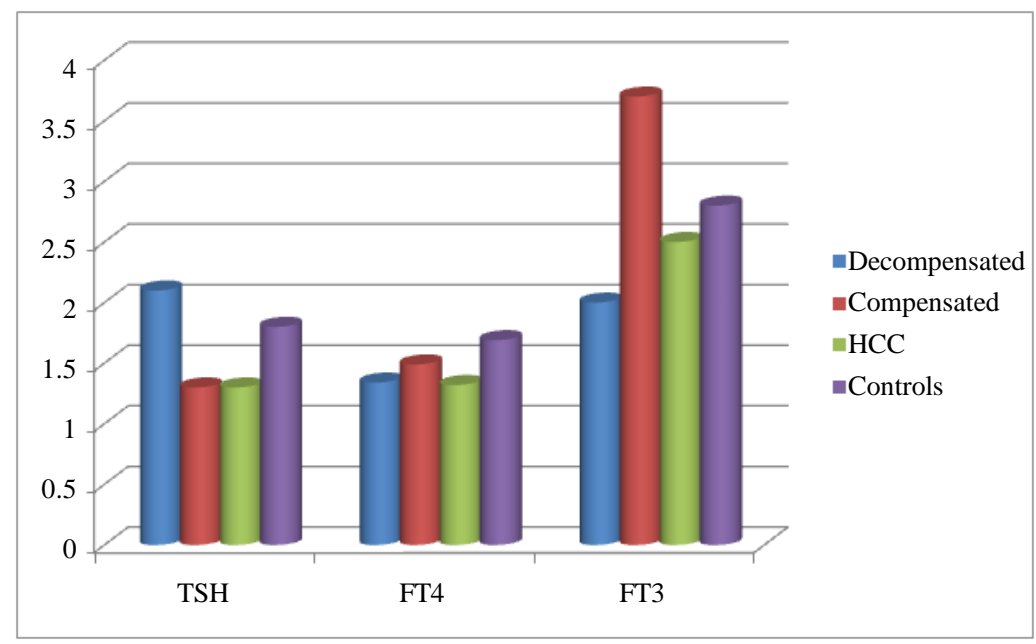

Figure 2. Comparison of the levels of TSH, FT3 and FT4 among various groups. FT3 was significantly higher in the compensated group versus other groups and significantly lower in the decompensated group by one way ANOVA test (pvalue $<0.001$ ). No statistically significant differences were noted in TSH and FT4 values among the various groups. 


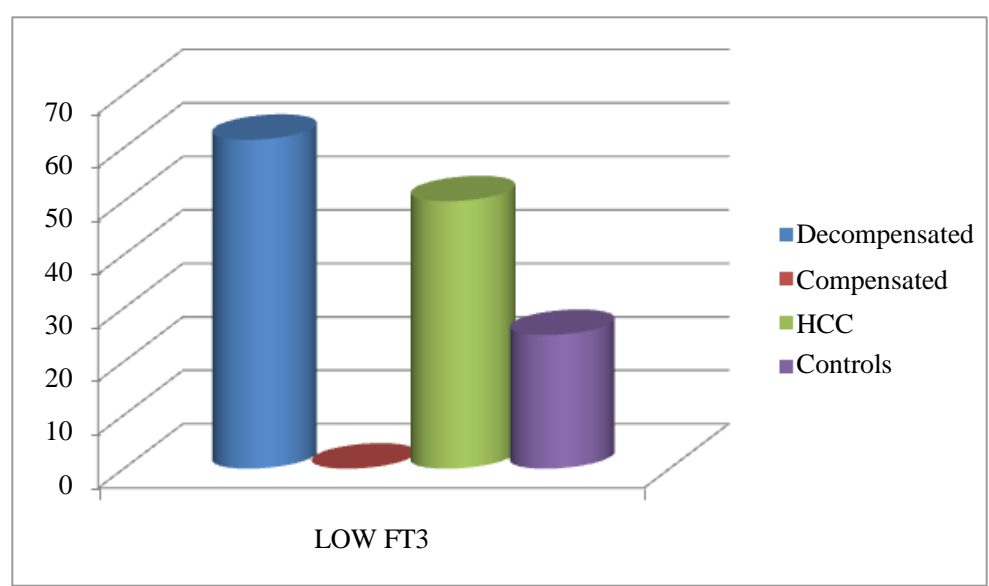

Figure 3. Percentage of subjects with low FT3 in each group. More than $60 \%$ of decompensated patients had low FT3, as compared to $50 \%$ of patients with HCC, and none of compensated patients. This difference was statistically significant ( $\mathrm{p}$-value $<0.001$ ).

Table 3. Comparison between the studied groups as regard thyroid profile categories.

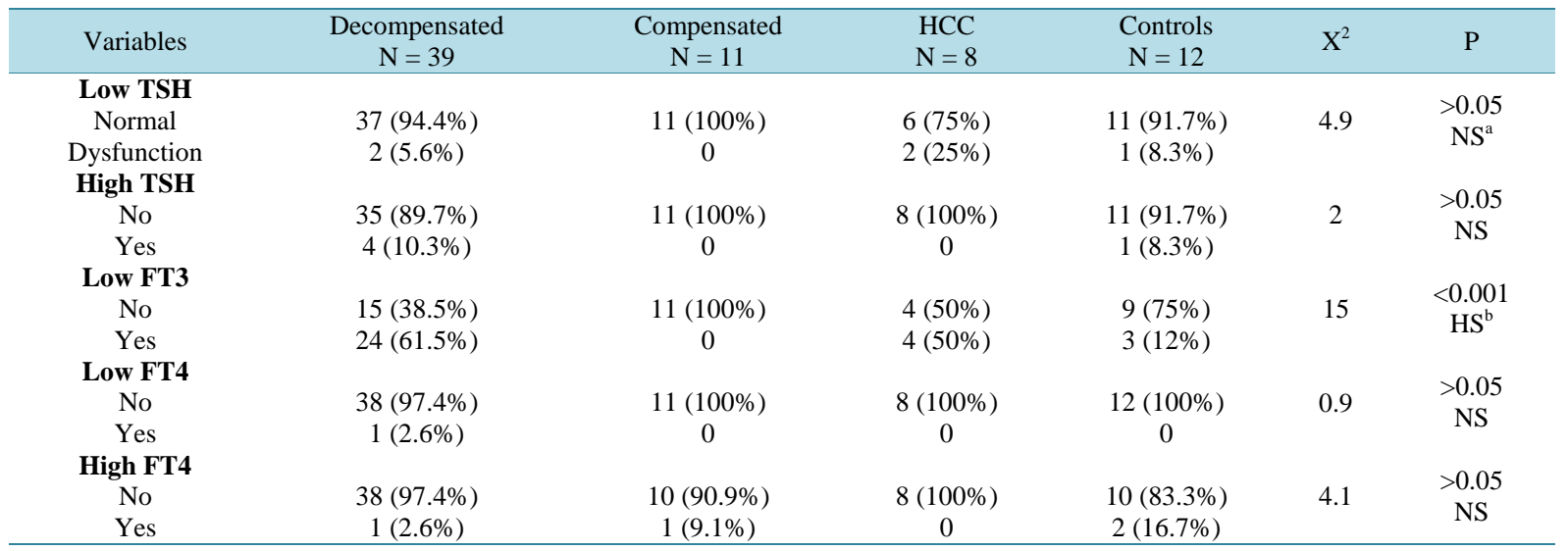

${ }^{\mathrm{a} N S}$ non-significant; ${ }^{\mathrm{b}} \mathrm{HS}$ highly significant.

The presence of ascites, male gender and hypoalbuminemia were found to be independently associated with the development of encephalopathy (p-value < 0.05) (Table 4).

Hypoalbuminemia and variceal bleeding were found to be independently associated with the development of ascites (p-value $<0.05$ ) (Table 5).

Low FT3, male gender, ulcer bleeding and encephalopathy were independently associated with the development of HCC (Table 6). This was evident after adjusting for compounding variables namely splenomegaly, ascites, SBP, encephalopathy, variceal bleeding, liver function tests and lipid profile. Of the 4 independent risk factors for HCC, low FT3 had the greatest contribution to its development, with a beta-coefficient 0.98 . This was followed by male gender with a beta-coefficient 0.44 , then ulcer bleeding to a lesser degree and lastly encephalopathy.

\section{Discussion}

The thyroid function derangements found in this study may be attributed either to a true thyroid dysfunction associated with liver disease or the well established entity of "nonthyroidal illness syndrome (NTIS)" formerly known as sick euthyroid syndrome. These findings agree with previous studies that analysed thyroid dysfunction during critical illness. TSH levels are described to be commonly within the normal range in NTIS but may decrease in prolonged illness. 


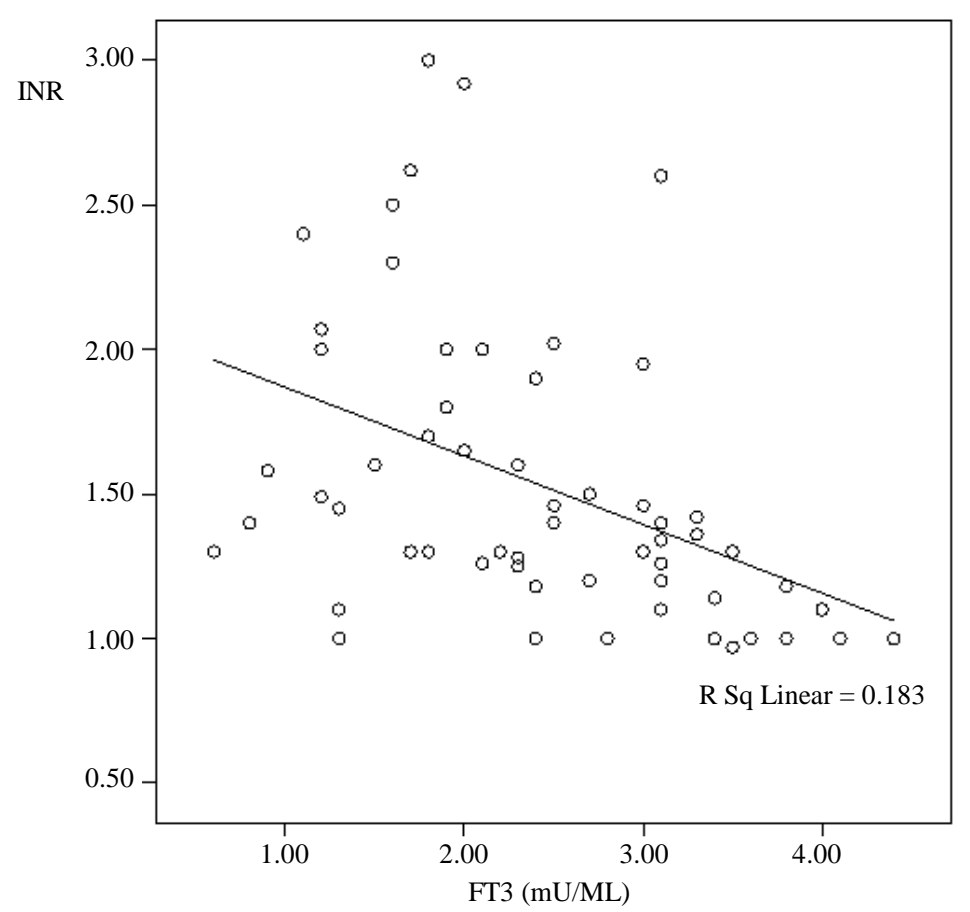

Figure 4. Scatter diagram showing correlation between FT3 and INR among all patients. FT3 shows a significant negative correlation with INR, correlation coefficient -0.30 , p-value $<0.05$.

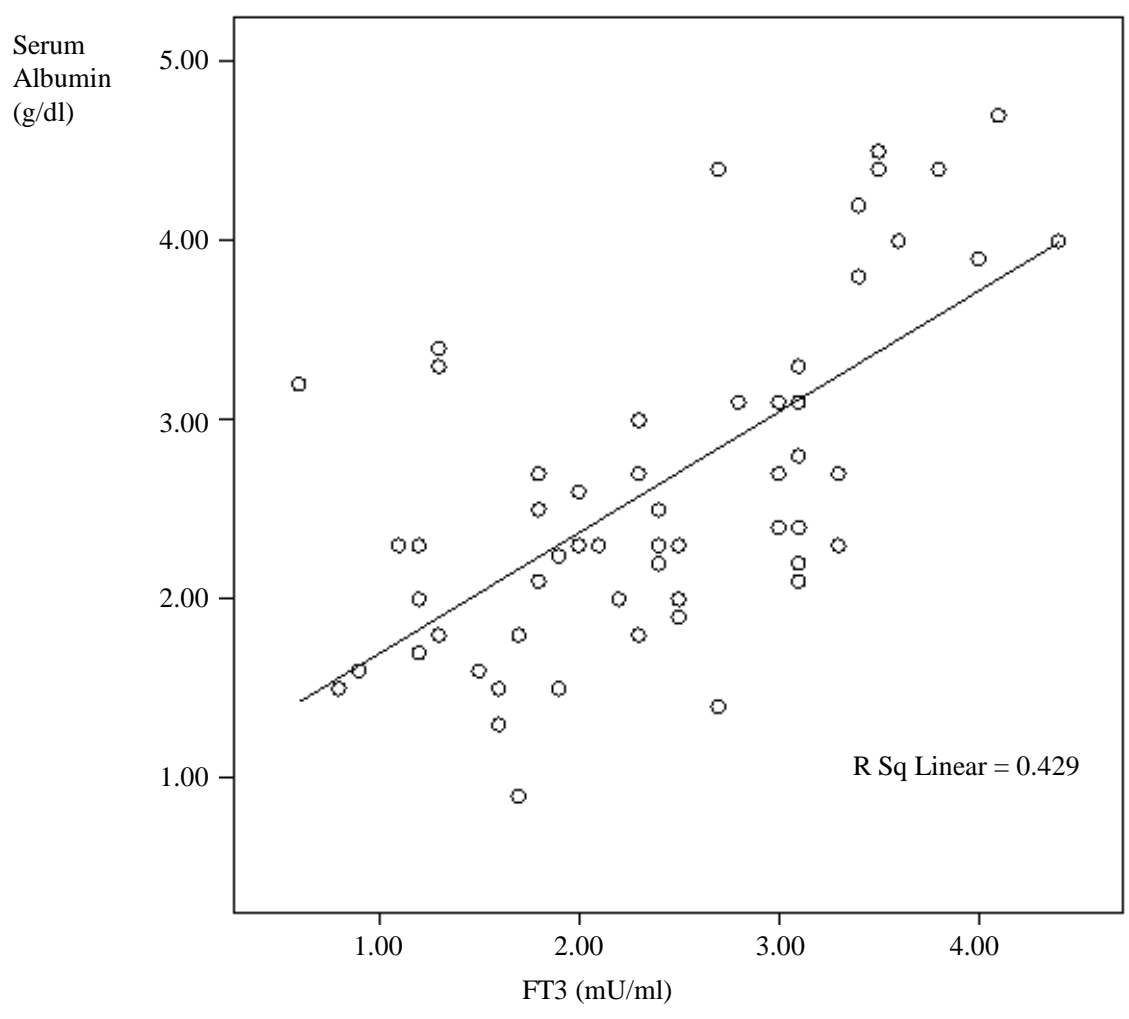

Figure 5. Scatter diagram showing correlation between FT3 and albumin among all patients. FT3 shows a significant positive correlation with albumin, correlation coefficient 0.48 , p-value $<0.05$. 
Table 4. Multivariable analysis between all risk factors versus encephalopathy by logistic regression.

\begin{tabular}{cccc}
\hline Variables & Beta-coefficient & $\mathrm{P}$ & Odd's (95\% CI) $^{\mathrm{a}}$ \\
\hline Ascites & 0.33 & $<0.05$ & $1.02(-0.1-10.3)$ \\
Male gender & 0.19 & $<0.05$ & $1.05(-0.8-12.7)$ \\
Low albumin $(<3)$ & 0.12 & $<0.05$ & $1.01(-0.9-22.8)$ \\
\hline
\end{tabular}

${ }^{\mathrm{a}} \mathrm{CI}=$ confidence interval.

Table 5. Multivariable analysis between all risk factors versus ascites by logistic regression.

\begin{tabular}{cccc}
\hline Variables & Beta-coefficient & $\mathrm{P}$ & ${\text { Odd's (95\% CI })^{\mathrm{a}}}^{\text {L }}$ \\
\hline Low albumin $(<3)$ & 0.21 & $<0.05$ & $1.1(-0.5-19.7)$ \\
Variceal bleeding & 0.20 & $<0.05$ & $1.08(-0.3-12.8)$ \\
\hline
\end{tabular}

${ }^{\mathrm{a}} \mathrm{CI}=$ confidence interval.

Table 6. Multivariable analysis between all risk factors versus HCC by logistic regression.

\begin{tabular}{cccc}
\hline Variables & Beta-coefficient & $\mathrm{P}$ & ${\text { Odd's }(95 \% \mathrm{CI})^{\mathrm{a}}}^{\text {Low FT3 }}$ \\
Male gender & 0.98 & $<0.05$ & $1.1(0.3-8)$ \\
Ulcer bleeding & 0.44 & $<0.05$ & $1.07(-0.06-11.7)$ \\
Encephalopathy & 0.22 & $<0.05$ & $1.01(-0.4-13.6)$ \\
\hline
\end{tabular}

${ }^{\mathrm{a}} \mathrm{CI}=$ confidence interval.

A closer look at these abnormalities is required. In this study, patients with low TSH included 2 patients with decompensated liver cirrhosis. Both had low FT3 and normal FT4. The level of TSH was less than 0.3 but more than $0.05 \mathrm{mU} / \mathrm{ml}$. Only lower TSH levels are associated with true hyperthyroidism in some cases. Such patients fit the definition of NTIS, which is usually found in $60 \%-70 \%$ of critically ill patients [9].

Also, 2 patients with HCC had low TSH. One had TSH level $0.03 \mathrm{mU} / \mathrm{ml}$, a normal FT4 and low FT3. This can also be attributed to NTIS due to associated low FT3, but later follow up is mandatory due to associated low TSH levels. The other patient had TSH in the range $0.3-0.05(0.2 \mathrm{mU} / \mathrm{ml})$, normal FT3 and FT4. This pattern can also be explained by NTIS, although one control subject had almost the same pattern.

On the other hand, all three patients in this study with high TSH had decompensated liver disease. All three had low FT3, and a TSH level $<20 \mathrm{mU} / \mathrm{ml}$. This pattern is quite typical of NTIS. Some hospitalized patients have transient elevations in serum TSH concentrations (up to $20 \mathrm{mU} / \mathrm{L}$ ) during recovery from nonthyroidal illness. Few of these patients prove to have hypothyroidism when re-evaluated after recovery from their illness. Patients with serum TSH concentrations over $20 \mathrm{mU} / \mathrm{L}$ usually have permanent hypothyroidism [10]. Two of our patients had TSH $>10 \mathrm{mU} / \mathrm{ml}$ should require later follow up.

More importantly, the frequency of patients with FT3 lower than the normal range was significantly higher than control subjects. Low FT3 was found in $48 \%$ of patients with liver cirrhosis and 50\% of patients with HCC, as compared to only $12 \%$ in controls (p-value $<0.001$ ). These figures may reflect NTIS, but again, the difference in distribution of low FT3 among patients with liver cirrhosis is clinically relevant.

By analysis of the relative frequencies of abnormal thyroid function among various groups, the prevalence of low FT3 (below the lower limit) was highest in decompensated patients (61.5\%), followed by HCC patients (50\%) versus none of the compensated patients. This difference was statistically significant with a p-value < 0.001. Other thyroid function abnormalities were matching with frequencies in healthy controls.

Indeed, samples for thyroid function were collected from decompensated patients who required hospital admissions during an acute illness, in the form of SBP, encephalopathy or GIT haemorrhage. While compensated patients were sampled on an outpatient basis.

These results come in agreement with most previous studies. The majority of critically ill patients have low serum T3 concentrations, as do some outpatients during illness [11].

In addition to lower FT3 levels with clinical decompensation, FT3 also decreases with progression of liver 
cirrhosis and deterioration of liver function tests.

This association of low FT3 with hepatic decompensation and deterioration of liver functions was demonstrated in previous studies [12]-[14].

It is worthy of note that the single mortality in this study was a patient with the second lowest FT3 level-0.8 $\mathrm{pg} / \mathrm{ml}$ (normal 2.4 - 4.2). The patient had a PC 60\%, albumin 1.5, ascites and presented with hepatic encephalopathy. Other patients with hepatic encephalopathy (a total of 16 patients) survived. Hence, the prognostic value of FT3 level, not only in liver disease severity, but also the outcome in acute complications.

The low total and free T3 levels may be regarded as an adaptive hypothyroid state that serves to reduce the basal metabolic rate within hepatocytes and preserve liver function and total body protein stores. A study in cirrhotic patients showed that the onset of hypothyroidism from intrinsic thyroid disease of various etiologies during cirrhosis resulted in a biochemical improvement in liver function (e.g. coagulation profiles) as compared to cirrhotic controls [15]. Hypothyroidism has also been associated with lesser degrees of decompensation in cirrhosis [16].

The independent association between low FT3 and HCC in the regression model suggests a true association between thyroid dysfunction and HCC, rather than a simple reflection of deterioration in liver functions. Firstly, it had the greatest impact on HCC development. Two complications, ulcer bleeding and encephalopathy had only a minor role. Lastly, all HCC patients were outpatients and not acutely ill. Several previous studies had analysed a similar association with overt hypothyroidism [17] [18].

A possible role for T3 in suppression of HCC cell invasiveness was suggested in a previous study on HCC cell lines [7]. Thus, the independent association of low FT3 with HCC in this study may suggest that the implication of low T3 is more than an index of hepatic disease and HCC. Prolonged depression of FT3 serum levels in patients with liver cirrhosis appears to have a pathogenic role in HCC development.

\section{Limitations and Further Investigations}

In statistical analysis, the impact of TSH and FT4 derangements as well as risk factors for mortality was impossible due to their rare prevalence among the studied sample. Concerning analysis of HCC risk factors, lipid profile was taken into consideration besides clinical and laboratory measures of liver status. Further studies comprising larger samples should adjust for other well established risk factors for HCC as smoking, obesity and diabetes.

The authors conclude that low FT3 is common among patients with decompensated liver cirrhosis and HCC. FT3 shows a significant negative correlation with severity of liver disease and deterioration of liver function. It may serve also as a prognostic factor in critically ill cirrhotic patients. Also, low FT3 shows a significant independent association with HCC.

The authors suggest that serum FT3 level be an index for liver status, as well as a marker for prognosis in hospitalized liver cirrhosis patients. It may be used as a risk factor and a marker for HCC development.

\section{Acknowledgements}

We would like to acknowledge our great Kasr Alainy Hospital, and its workers, nurses and staff members, for all support and help in this study and throughout our careers.

\section{Funding}

Authors received no funding for this study.

\section{Declaration of interest}

The authors report no conflicts of interest. The authors alone are responsible for the content and writing of the paper.

\section{References}

[1] Malik, R. and Hodgson, H. (2002) The Relationship between the Thyroid Gland and the Liver. QJM, 95, 559-569. http://dx.doi.org/10.1093/qjmed/95.9.559

[2] Guven, K., Kelestimur, F. and Yucesoy, M.(1993) Thyroid Function Tests in Non-Alcoholic Cirrhotic Patients with Hepatic Encephalopathy. The European Journal of Medicine, 2, 83-85. 
[3] Van Thiel, D.H., Udani, M., Schade, R.R., Sanghvi, A. and Starzl, T.E. (1985) Prognostic Value of Thyroid Hormone Levels in Patients Evaluated for Liver Transplantation. Hepatology, 5, 862-866. http://dx.doi.org/10.1002/hep.1840050526

[4] Güven, K., Kelestimur, F. and Yücesoy, M. (1993) Thyroid Function Tests in Non-Alcoholic Cirrhotic Patients with Hepatic Encephalopathy. The European Journal of Medicine, 2, 83-85.

[5] Pucci, E., Chiovato, L. and Pinchera, A. (2000) Thyroid and Lipid Metabolism. International Journal of Obesity and Related Metabolic Disorders, 24, S109-S112. http://dx.doi.org/10.1038/sj.ijo.0801292

[6] Liangpunsakul, S. and Chalasani, N. (2003) Is Hypothyroidism a Risk Factor for Non-Alcoholic Steatohepatitis? Journal of Clinical Gastroenterology, 37, 340-343. http://dx.doi.org/10.1097/00004836-200310000-00014

[7] Hassan, M.M., Kaseb, A., Li, D., et al. (2009) Association between Hypothyroidism and Hepatocellular Carcinoma: A Case-Control Study in the United States. Hepatology, 49, 1563-1570. http://dx.doi.org/10.1002/hep.22793

[8] Baloch, Z., Carayon, P., Conte-Devolx, B., et al. (2003) Laboratory Medicine Practice Guidelines. Laboratory Support for the Diagnosis and Monitoring of Thyroid Disease. Thyroid, 13, 3-126. http://dx.doi.org/10.1089/105072503321086962

[9] Economidou, F., Douka, E., Tzanela, M., Nanas, S. and Kotanidou, A. (2011) Thyroid Function during Critical Illness. Hormones, 10, 117-124. http://dx.doi.org/10.14310/horm.2002.1301

[10] Burman, K.D. and Wartofsky, L. (2001) Endocrine and Metabolic Dysfunction Syndromes in the Critically Ill: Thyroid Function in the Intensive Care Unit Setting. Critical Care Clinics, 17, 43-57. http://dx.doi.org/10.1016/S0749-0704(05)70151-2

[11] Peeters, R.P., Wouters, P.J., van Toor, H., Kaptein, E., Visser, T.J., Van den Berghe, G., et al. (2005) Serum 3,3’,5’Triiodothyronine (rT3) and 3,5,3'-Triiodothyronine/rT3 Are Prognostic Markers in Critically Ill Patients and Are Associated with Postmortem Tissue Deiodinase Activities. The Journal of Clinical Endocrinology \& Metabolism, 90, 4559-4565. http://dx.doi.org/10.1210/jc.2005-0535

[12] Mansour-Ghanaei, F., Mehrdad, M., Mortazavi, S., et al. (2012) Decreased Serum Total T3 Level in Hepatitis B and C Related Cirrhosis by Severity of Liver Damage. Annals of Hepatology, 11, 667-671.

[13] L'age, M., Meinhold, H., Wenzel, K.W. and Schleusener, H. (1980) Relations between Serum Levels of TSH, TBG, T4, T3, rT3 and Various Histologically Classified Chronic Liver Diseases. Journal of Endocrinological Investigation, 3, 379-383. http://dx.doi.org/10.1007/BF03349374

[14] Faber, J., Thomsen, H.F., Lumholtz, I.B., Kirkegaard, C., Siersbaek-Nielsen, K. and Friis, T. (1981) Kinetic Studies of Thyroxine, 3,5,3'-Triiodothyronine, 3,3,5'-Triiodothyronine, 3',5'-Diiodothyronine, 3,3'-Diiodothyronine, and 3'-Monoiodothyronine in Patients with Liver Cirrhosis. The Journal of Clinical Endocrinology \& Metabolism, 53, 978-984. http://dx.doi.org/10.1210/jcem-53-5-978

[15] Oren, R., Sikuler, E., Wong, F., Blendis, L.M. and Halpern, Z. (2000) The Effects of Hypothyroidism on Liver Status of Cirrhotic Patients. Journal of Clinical Gastroenterology, 31, 162-163. http://dx.doi.org/10.1097/00004836-200009000-00016

[16] Oren, R., Brill, S., Dotan, I. and Halpern, Z. (1998) Liver Function in Cirrhotic Patients in the Euthyroid versus the Hypothyroid State. Journal of Clinical Gastroenterology, 27, 339-341. http://dx.doi.org/10.1097/00004836-199812000-00012

[17] Reddy, A., Dash, C., Leerapun, A., Mettler, T.A., Stadheim, L.M., Konstantinos, N., et al. (2007) A Possible Risk Factor for Liver Cancer in Patients with No Known Underlying Cause of Liver Disease. Clinical Gastroenterology and Hepatology, 5, 118-123. http://dx.doi.org/10.1016/j.cgh.2006.07.011

[18] Lin, K.-H., Lin, Y.-W., Lee, H.-F., Liu, W.-L., Chen, S.-T., Chang, K.S.S. and Cheng, S.-Y. (1995) Increased Invasive Activity of Human Hepatocellular Carcinoma Cells Is Associated with an Overexpression of Thyroid Hormone $\beta 1$ Nuclear Receptor and Low Expression of the Anti-Metastatic nm23 gene. Cancer Letters, 98, 89-95. http://dx.doi.org/10.1016/S0304-3835(06)80015-7 http://dx.doi.org/10.1016/0304-3835(95)04000-T 